\title{
Comparison of mobile and clinical EEG sensors through resting state simultaneous data collection
}

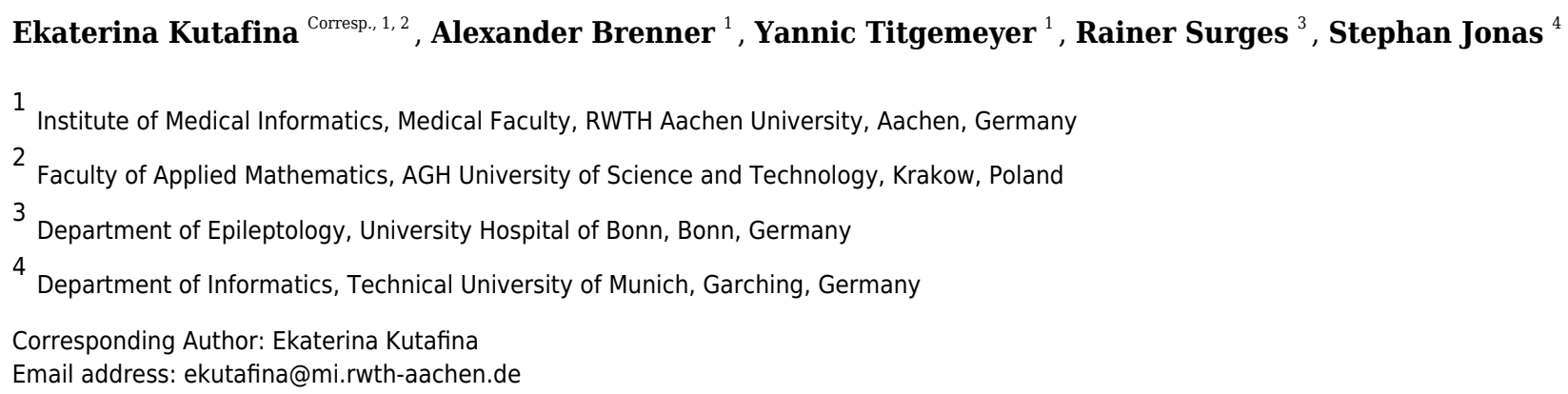

Development of mobile sensors brings new opportunities to medical research. In particular, mobile electroencephalography (EEG) devices can be potentially used in low cost screening for epilepsy and other neurological and psychiatric disorders. The necessary condition for such applications is thoughtful validation in the specific medical context. As part of validation and quality assurance, we develop a computer-based analysis pipeline, which aims to compare the EEG signal acquired by a mobile EEG device to the one collected by a medically approved clinical-grade EEG device. Both signals are recorded simultaneously during 30 minutes long sessions in resting state. The data are collected from 22 patients with epileptiform abnormalities in EEG. In order to compare two multichannel EEG signals with differently placed references and electrodes, a novel data processing pipeline is proposed. It allows deriving matching pairs of time series which are suitable for similarity assessment through Pearson correlation. The average correlation of 0.64 is achieved on a test dataset, which can be considered a promising result, taking the positions shift due to the simultaneous electrode placement into account. 
1 Comparison of mobile and clinical EEG sensors through resting state simultaneous data collection.

3

4 Ekaterina Kutafina ${ }^{1,2}$, Alexander Brenner ${ }^{1}$, Yannic Titgemeyer ${ }^{1}$, Rainer Surges ${ }^{3}$, Stephan

5 Michael Jonas ${ }^{4}$

6

7 'Institute of Medical Informatics, Medical Faculty, RWTH Aachen University, Pauwelsstr. 30,

852074 Aachen, Germany

$9 \quad{ }^{2}$ Faculty of Applied Mathematics, AGH University of Science and Technology, Krakow, Poland

$10{ }^{3}$ Department of Epileptology, University Hospital of Bonn, Bonn, Germany

$11{ }^{4}$ Department of Informatics, Technical University of Munich, Garching, Germany

12

13 Corresponding Author:

14 Ekaterina Kutafina

15 Pauwelsstrasse 30, 52057 Aachen, Germany

16 email: ekutafina@ukaachen.de

17

18

19

20

21

22

23

24

25

26

27

28

29

30

31

32

33

34

35

36

37

38

39 
40

41

42

43

44

45

46

47

48

49

50

51

52

53

54

55

56

57

58

59

60

61

62

63

64

65

66

67

68

69

70

71

72

73

74

75

76

77

Abstract: Development of mobile sensors brings new opportunities to medical research. In particular, mobile electroencephalography (EEG) devices can be potentially used in low cost screening for epilepsy and other neurological and psychiatric disorders. The necessary condition for such applications is thoughtful validation in the specific medical context. As part of validation and quality assurance, we develop a computer-based analysis pipeline, which aims to compare the EEG signal acquired by a mobile EEG device to the one collected by a medically approved clinical-grade EEG device. Both signals are recorded simultaneously during 30 minutes long sessions in resting state. The data are collected from 22 patients with epileptiform abnormalities in EEG. In order to compare two multichannel EEG signals with differently placed references and electrodes, a novel data processing pipeline is proposed. It allows deriving matching pairs of time series which are suitable for similarity assessment through Pearson correlation. The average correlation of 0.64 is achieved on a test dataset, which can be considered a promising result, taking the positions shift due to the simultaneous electrode placement into account.

\section{Introduction}

Fast development of mobile and wearable sensors introduces new opportunities for field-trials and long-term monitoring in many research and clinical areas. However, these novel sensors require a rigorous validation, particularly when being used in clinical applications. The methods of validation can differ greatly depending on the type of the sensor and the purpose of the collected data. In this manuscript we focus on the comparison of a novel, wearable electroencephalography (EEG) device to a clinical equivalent with the aim of sensor validation.

In principle, two main approaches to quantitative EEG validation can be found in the literature:

Technical validation includes an assessment of electrical characteristics of the device or just the electrodes. This approach is often chosen by sensor developers or researchers as an initial validation. For example, Liao et al. (1) report the comparison of dry electrodes impedances to impedances of standard clinical wet electrodes. Generated signals of fixed frequency (2) can be used for a spectral analysis of the record. Play-back $(1,3)$ is a technique where brain signal is recorded with the gold standard device and then re-played and recorded by the device in question. In vivo experiments are challenging, as the signal cannot be recorded at the same time and place with multiple devices. There are two possible solutions (3): "same time different 
78 place", where several sensors are placed as close to each other as possible and data collected

79 simultaneously; and "same place different time", where the recordings are being performed

80 subsequently at the exact same positions. These methods are also referred to as parallel and serial

81 (4). In the first case, the recorded signals can be compared through visual analysis $(4,5)$, cross

82 correlation $(1,2,4-6)$, spectral features $(7)$, mutual information $(8,9)$ or other numerical features.

83 In case of serial recordings, the options for quality assessment are more limited due to time-

84 dependent changes in brain activity.

85

86

87

88

89

90

91

92

93

94

95

96

97

98

99

100

101

102

103

104

105

106

107

108

109

110

111

112

113

114

115

116

117

Another possibility is a validation in a given experimental context. It can be done to answer the question if the new device is suitable for a certain type of research or medical purposes. Here, the choice of possible tests is highly dependent on the application area, but typically the data are collected under well controlled conditions. Both parallel and serial setups can be used. For instance, the Emotiv Epoc mobile EEG has been investigated by Badcock et al. (10). The authors compare statistical features of auditory Event Related Potentials (ERP) captured in parallel experiments by the mobile and a research-grade device. Melnik et al. (2017) (11) used six different ERP paradigms and serial approach to study the variances caused by multiple sessions, subjects and devices. Another common option is an experiment when the subjects are instructed to have their eyes open and closed for a certain time interval. This allows comparing the EEG alpha band powers (2).

While all the above approaches are necessary and valid in a research and industrial environment, further validation is required prior to clinical use. Clinical validation proves that a device is capable of performing similar or better than an existing clinical gold standard under real-world conditions. Unfortunately, all described methods require performing the data collection under carefully controlled conditions, which is not always possible in a clinical environment. Some earlier publications $(12,20)$ proposed a solution based on professional human assessment, however this approach brings a number of problems, such as subjective judgement and high variation of the scores (12). Therefore, an objective, computer-based way of comparison is needed.

The present work aims to answer the following research question: How to compare two multichannel EEG system using a fully computer-based data analysis pipeline and simultaneously recorded data under the following limitations:

- No hardware modification can be done. In particular, the referencing electrodes are placed according to the respective design of the devices.

- No special external stimuli can be introduced, which limits statistical analysis of the signal responses.

The developed pipeline is investigated using simultaneously acquired data from a mobile and clinical EEG during a clinical study at the RWTH Aachen University Hospital. 


\section{Methods}

\section{Study design}

120

121

122

123

124

125

126

127

128

129

130

131

132

133

134

135

136

137

138

139

140

141

142

143

144

145

146

147

148

149

150

151

152

153

154

155

Resting state EEG signals were collected simultaneously with a clinical and mobile EEG devices from a gender-balanced group of 22 patients admitted to the hospital for epilepsy diagnostics. Average age of the patients was $40.2 \pm 15$ years and the more detailed demographics can be found in Tab 1. All patients were fluent in German language and signed the informed consent. The study was approved by the Ethical Board of Uniklinik RWTH Aachen (EK150-17 3.7.2017) and registered prospectively (DRKS-ID: DRKS00012424). Written consent was obtained from each participant. The primary goal of the study was to assess the quality of the mobile EEG device for epileptiform abnormality detection and the results of the human-based evaluation can be found in (12).

The clinical grade data were collected with Brain Quick Plus Evolution by Micromed, which is currently used as a standard device at Epileptology Section at Uniklinik RWTH (Aachen, Germany) where the recordings were conducted. This model has 21 EEG electrodes, ground electrode G1 (positioned between Fz and Cz on the left side) and reference electrode G2 (positioned between $\mathrm{Fz}$ and $\mathrm{Cz}$ on the right side).

The electrodes are placed according to the 10-20 system (13) (see also Fig. 1a). The data are sampled at $256 \mathrm{~Hz} .0 .18 \mathrm{~Hz}$ high-pass filtering is performed in the amplifier. During patients' hospital stay they were undergoing continuous EEG monitoring for several days, with simultaneous collection of video data. Therefore we use the abbreviation vEEG for the clinical EEG further on. The video data were not used in our study. Approximately every 3 hours of EEG records are saved in a separate file and exported in the European Data Format (.EDF files).

For the mobile data collection, the Epoc Emotiv device was used (mEEG). This device was originally marketed as a mobile brain-computer interface (BCI) sensor, but gained a lot of interest from the research community. Previous works showed that this particular device is able to capture EEG potentials and is promising in research context $(10,11)$. Despite a somewhat limited coverage of the cerebral cortex, the price range and number of electrodes (14, CMS/DRL references) made it a potentially useful tool for clinicians and researchers. The electrode positions are fixed due to a rigid plastic frame and approximate the 10-20 system (see Fig. 1a). Sampling rate of the device used in the study is $128 \mathrm{~Hz}$.

In the experiment Epoc Emotiv was mounted while the clinical EEG device was already in use. mEEG was recorded for approximately 30 minutes within a single recording session. During these 30 minutes the patients were asked to limit their movements, as Emotiv Epoc device is highly prone to movement artifacts. At the beginning of the mobile data collection, the 
156 participants were asked to blink strongly several times in a row. This was done to facilitate the

157 later data alignment.

158 The electrodes were placed as close as possible to the vEEG electrodes, at the same time

159 minding the distance enforced by the glue used to attach vEEG electrodes.

160 The stiff frame of $\mathrm{mEEG}$ presented an additional challenge. After the mounting of $\mathrm{mEEG}$ a

161 sketch of the placement was made (see Fig. 1a for an example).

162 Fig. 2 shows an illustrative fragment of the same EEG fragment, recorded on both video (a) and

163 mobile (b) devices. An epileptiform abnormalities (spike-slow waves) are visible in both

164 versions.

165 Before any further processing, the data were pseudonymized and only the session's number was 166 used to keep track of the files.

167 Three patients' data were discarded from the trial. One set because of the mobile recording 168 software failure, one because of the failure of reference electrode in the clinical EEG, and the last 169 one because of cell phone usage by the patient resulting in strong artifacts.

\section{Software}

171 Data collection was performed with BrainLab (14). Data analysis was done with MATLAB 172 R2017b (The Mathworks, Natick, MA, USA) and EEGLAB v.14 (15).

\section{Data preprocessing}

174 The raw EEG data files have different length, sampling rates and electrode placements and, 175 therefore, require several preparation steps before the comparison between mobile and clinical 176 signals can be done. The pipeline presented below allows for constructing aligned pairs of signal 177 vectors, ready for further statistical analysis.

Initial pruning of clinical EEG data

180

181

182 183

184 185

The duration of the vEEG and mEEG signal is 3 hours and 30 minutes respectively. Based on the blinking and the marked time of the mEEG data collection, approximately 30 minutes of vEEG is cut (see Fig. 3). It is not yet perfectly aligned with the mEEG data, but the shift is within 15 sec.

\section{Selection of corresponding electrodes between vEEG and mEEG.}

The two considered EEG systems are differently referenced. Therefore, in order to be able to compare the data, we propose a shift to bipolar referencing, defined individually for each patient based on the specific relative electrode positions (see Fig. 1a). The couples of mobile and video electrodes placed directly next to each other (as close as the glue circle allows) are listed. For 
190

191

192

193

194

195

196

197

198

199

200

201

202

203

204

205

206

207

208

209

210

211

212

213

214

215

216

217

218

219

220

221

222

223

224

225

226

example, the following couples are chosen (Fig. 1b): mF3-vF3 and mT8-vT4. The set of two corresponding pairs of electrodes will be called a quadruple. The list of all possible electrode quadruples (e.g. [mF3, mT8, vF3, vT4], with $\mathrm{m}^{*}$ and $\mathrm{v}^{*}$ being electrodes from the $\mathrm{mEEG}$ and vEEG respectively) is created individually for each patient (Fig. 3). Some quadruples will later correspond to a pair of EEG vectors which we expect to present similarities.

\section{Choose well-aligned data vectors}

The initial choice of quadruples does not guarantee the spatial alignment. In order to assure such alignment, only the pairs which lie far enough from each other will be considered. For example, if we take a signal described by mFC5-mF3 difference and vC3-vF3 (Fig.1), we can see that the two vectors are far from being well aligned and it is a direct consequence of the spatial proximity of two matched pairs. In order to avoid arbitrary decisions regarding the good or bad alignment we have introduced a decision rule as follows.

a) The video electrodes are put on a grid (see Fig.4, the sides of the squares are assumed to have unit length) and then the Manhattan distance between each pair of electrodes is computed. The distances can take values from zero (from a given electrode to itself), up to six. Electrodes positioned from four to six steps from each other are considered to be sufficiently distant. The reference electrode is assumed to be placed exactly in the middle of the Fz-F4-C4-Cz square, and Manhattan distance from $\mathrm{Cz}$ to $\mathrm{G} 2$ equals to $0.5+0.5=1$.

b) For each participant the quadruples with video electrodes separated by Manhattan distance of 4 and higher are taken. Ultimately, 361 quadruples out of 876 possible are chosen.

\section{Data cleaning and extraction of quadruples}

Due to the relatively low signal-to-noise ratio, it is essential to reduce the noise before any comparison is made. Additionally, the data need to be synchronized in time and pruned to the same vector size. Therefore, the following data cleaning pipeline was implemented (see Fig. 5 for the visualisation):

1) The EEGLAB function pop_rejchan is used to detect corrupted channels in all files. The quadruples containing such channels are removed from the list.

2) Low pass filtering at $f q \_l o w p a s s \mathrm{~Hz}$ (the parameter $f q \_l o w p a s s$ depends on the chosen frequency band).

3) Downsampling vEEG to a sampling rate of $128 \mathrm{~Hz}$, to match the lower mEEG sampling rate.

4) High pass filtering at $f q$ _highpass $\mathrm{Hz}$ (similarly to $f q_{-}$lowpass, the parameter $f q$ _highpass depends on the chosen frequency band). 
227

228

229

230

231

232

233

234

235

236

237

238

239

240

241

242

243

244

245

246

247

248

249

250

251

252

253

254

255

256

257

258

259

260

261

262

5) For each patient, the perc-th percentiles of absolute values of the amplitudes are computed for $\mathrm{mEEG}$ and vEEG separately, and the corresponding raw data are divided by the resulting value. This normalization allows to avoid the problem of different scales of $\mathrm{mEEG}$ and vEEG.

As an output of the described process, we obtain filtered, normalized and roughly time-aligned mobile and clinical EEG data sets for each patient. Additionally the corrupted channels are eliminated and an individual list of channel quadruples to be used for further re-referencing is stored.

\section{Processing of single quadruples}

More precise time alignment and artifact removal is done for individual quadruples (e.g. [mF3, $\mathrm{mT} 8, \mathrm{vF} 3, \mathrm{vT} 4]$ as illustrated on Fig. 1b) of specific patients. The resulting vectors can be compared through Pearson correlation.

\section{Artifact detection for a single channel}

In order to capture short-term signal disturbances, we construct an adjusted procedure for artifact detection:

1) First, threshold AmThresh is fixed and all data with absolute value exceeding AmThresh is marked as $\mathrm{NaN}$ (not a number). The data points are not removed to allow later time synchronization. It should be noted, that previous data normalization makes it possible to choose for a common single threshold for both $\mathrm{mEEG}$ and $\mathrm{vEEG}$ records.

2) Next, non-overlapping intervals of length WinLength are taken, average of amplitudes' absolute values is computed and the whole window is marked as $\mathrm{NaN}$ if this average exceeds the parameter AmThreshWin.

3) Artifact index is computed by dividing the length of the "corrupted" data (marked as $\mathrm{NaN}$ by the total data vector length.

4) If Artifact index exceeds $70 \%$, all quadruples including this channel are removed from the list.

The described procedure is subsequently applied for all four time series involved in the quadruple.

Remark 1. One of the most common procedures for EEG analysis is the removal of eye blinking artifacts. Here, it was decided against this removal, because the locations typically known for strong eye artifacts usually are not involved in the analysis. Additionally, such artifacts are a normal part of the EEG signal, and as such should manifest similarly in mEEG and vEEG signals. One may argue, that high amplitude of such signals may unproportionally influence the linear correlation, but since they are not very prominent in this particular data they were neglected. Similarly, ECG artifacts were not significant in the considered data. 
263 Re-reference of quadruples.

264 We mathematically re-reference the electrodes within each quadruple (through pairwise

265 subtraction) to eliminate the effect of the global reference (e.g. quadruple [mF3, mT8, vF3, vT4]

266 equivalent to [mF3-mRef, mT8-mRef, vF3-vRef, vT4-vRef] after re-referencing results in a pair

267 [mF3-vF3, mT8-vT4]). This subtraction can be thought of as bipolar (BP) re-referencing.

268 Proximity between corresponding video and mobile electrodes in combination with well-aligned

269 bipolar vectors should result in similarity of those vectors. Therefore, the time series $\mathrm{mF} 3-\mathrm{mT} 8$

270 is expected to show strong similarities with time series vF3-vT4. Note, that both time series may

271 contain $\mathrm{NaN}$ terms propagated from the artifact removal procedure and that the bipolar

272 referencing is different for each patient and is based on patient-specific relative electrode

273 placement. Similarly, the number of quadruples may vary.

274 Fine aligning

275 At this stage, MATLAB function lag can be used to find a time shift between the video and

276 mobile data. In order to check how the signal shifts progress in time and capture possible

277 (non)linear drift all signals were divided into 4 equal parts. The lags between mobile and video

278 signals were computed for each quadruplet and the resulting median per patient. The results are

279 presented in table A1, and suggest, that the drift in this particular experiment can be neglected.

280 What is important to account for, is the fact, that finding the correct lag is only possible if the

281 data has a certain minimal signal-to-noise ratio, otherwise all correlations are close to zero and

282 the time shift is set to a completely wrong value by the algorithm. For one recording session it is

283 possible that the lags slightly vary between quadruples, but typically the differences are within 1-

2842 data points. Therefore the lags are first computed for all quadruples of a given patient, then the

285 majority vote is used to set the same time shift to all combinations, allowing a fluctuation of \pm 5

286 data points to compensate for differences in device-specific recording order of the channels.

287

288 Remark 2. If the frequency band chosen for filtering is too narrow, errors might occur in the lag

289 computation. Therefore it is recommended to pre-compute and save lags for a wider band.

290

291

Finally, the longer vEEG vectors are pruned to match the length of the mEEG vectors and the

292

293 time stamps from the $\mathrm{mEEG}$ data are added. NaN data points from each single time series in the quadruple propagated to the bipolar re-referencing. After the aligning, all data segments where

294

295 either of the two bipolar time series contained NaN were removed (see Fig. 6).

Finally, Pearson linear correlation for the given quadruple is computed and stored.

296

297

298

299

\section{Aggregating data for multiple patients}

For each patient and fixed set of the parameters, the table containing all quadruples and their corresponding correlation coefficients is built. The patient average and the grand average across all patients can be reported. Due to the skewed data distribution, Fisher z-transform is used to 
300

301

302

303

304

305

306

307

308

309

310

311

312

313

314

315

316

317

318

319

320

321

322

323

324

325

326

327

328

329

330

331

332

333

334

335

336

normalize the data before computing the average. Afterwards the inverse transform is performed to return to the original scale. Additionally, the data loss resulting from all processing steps is tracked.

\section{Optimization and parameters choice}

Let us notice that there are 6 parameters which we can choose in the above described procedure: fq_lowpass, fq_highpass, perc, AmThresh, WinLength and AmThreshWin. A procedure for establishing parameters values consists of several steps. The parameters are optimized for all patients at once resulting in only one set of parameters that is used for all further calculations on all patients. Except for the lag between vEEG and $\mathrm{mEEG}$, no patient-specific parameter is necessary.

First, we fix the default filtering parameters $f q_{-}$lowpass and fq_highpass based on the estimation of the usable spectrum of the data. In principle the frequency parameters can be chosen freely, depending on which part of the spectrum the correlation is of interest. For instance, we will argue later, that alpha band $(7.5-12.5 \mathrm{~Hz})$ shows the best correlation. Nevertheless, in order to find the broadest default frequency band reasonable for our research, the average cross-spectrum of the data is computed and thresholding is made to fix the initial fq_lowpass and fq highpass values (Fig. 7). This cross-spectrum is computed in a procedure similar to the above described correlation computation, with only the initial high-pass filtering of mEEG at $0.5 \mathrm{~Hz}$. Other filtering, normalization and artifact removal are skipped for the estimation of the filter values. Second, a set of discrete values for perc, AmThresh, WinLength, AmThreshWin is chosen. For each parameter combination and patient, the correlation tables are computed.

Third, the data are randomly divided into two subsets of 14 training and 5 test records. These subsets are fixed and no cross-validation is performed. The parameter set with the best resulting grand average correlation coefficient is established based on the first set and quasi-independently evaluated based on the second set.

\section{Correlations between vEEG electrodes (vEEG to vEEG correlation)}

As direct performance comparison, we also investigate the similarities within the vEEG signal and calculate the correlations. In this case, all neighbouring pairs of vEEG electrodes were defined (e.g. [vP4, vT6]), and underwent analogous artifact removal procedures. Instead of quadruples that are re-referenced between two electrodes from both vEEG and mEEG each, we use two vEEG electrodes and their reference electrode. Since both electrodes are referenced to the same reference electrode, this constructs a virtual quadruple (e.g., [G2-vP4, G2- vT6] with G2 being the reference electrode) and correlation between the two signals can be calculated. Similarly to the mobile-to-video case, too "short" quadruplets were rejected. Since here the centrally positioned electrode G2 is always involved, the distances may vary from 1 to 4 , and the step is now equals to 0.5 , as G2 is located between the regular electrodes. 3 was taken as a soft threshold here. 
337

338

339

340

341

342

343

344

345

346

347

348

349

350

351

352

353

354

355

356

357

358

359

360

361

362

363

364

365

366

367

368

369

370

371

In case of vEEG-mEEG, the difference between the computed correlation and perfect linear correlation value (1) is due to the following factors: a) devices quality differences, b) noise, c) spatial distances between two pairs within one quadruple (e.g. vF3 to mF3 and vT4 to mT8). In case of vEEG-vEEG correlation, the differences are due to a) noise and b) spatial distances between the two vEEG electrodes (e.g. vP4 to vT6). These distances are larger than in vEEG to mEEG pairs, but there is no spatial distance influencing the correlation for the reference electrode. If we assume that these differences give a comparable error and the noises are on the same level, then the differences in average correlation should reflect the differences in the devices quality.

An additional step is performed to make the comparison more sensible: since the distances chosen for mobile-video quadruples were [4,5,6] and for video-video quadruples $[3,4]$, we have computed additionally an average correlation for "medium" mobile-video quadruples of the length 3 and 4.

\section{Results}

\section{Establishing frequency interval for preprocessing}

In the first step the frequency band with the boundaries $f q$ highpass and $f q$ lowpass for the preprocessing is chosen for the analysis. The lower boundary $(f q$ highpass $)$ is set to $1 \mathrm{~Hz}$, which is sufficient to remove the drift from the EEG data. In order to set $f q_{-}$lowpass, the magnitudesquared coherence was calculated to assess which frequencies are useful for comparison (Fig. 7). The magnitude-squared coherence indicates the shared information between mEEG and vEEG in distinct frequency bands. A cut-off value of 0.1 was chosen, resulting in upper frequency band limit of $38 \mathrm{~Hz}$. This is in line with the commonly used frequency bands in EEG analysis (following (21), delta up to $3.5 \mathrm{~Hz}$, theta $3.5-7.5 \mathrm{~Hz}$, alpha $7.5-12.5 \mathrm{~Hz}$, beta $12.5-30 \mathrm{~Hz}$ and gamma $>30 \mathrm{~Hz})$. Recent research also shows the importance of higher gamma $(>50 \mathrm{~Hz})$ in certain application contexts $(16,17)$, but the chosen interval of $1-38 \mathrm{~Hz}$ is sufficient for the basic neurologic assessment.

\section{Parameter optimization}

For the chosen frequency band $(1-38 \mathrm{~Hz})$, we consider the following discrete values of the parameters:

perc $=\{80 \%, 85 \%, 90 \%, 95 \%, 97 \%, 99 \%\}$, AmThresh $=\{1,1.5,2.0,3.0,4.0,5.0,6.0,7.0,8,9,10\}$, AmThreshWin $=\{0.5,1.0,1.2,1.5,2,2.5\}$, WinLength $=\{15,50,75,100,200,250\}$. 
372

373 For all possible parameter combinations, the average correlation was computed on the randomly

374

375

376

377 perc $=85 \%$,

378 AmThresh=8.0,

379 WinLength $=100$,

380 AmThreshWin=1.5.

381

382

383

384

385

386

387

388

389

390

391

392

393

394

395

396

397

398

399

400

401

402

403

404

405

As a boundary condition, the amount of data retained needed to be $70 \%$ on the train data.

\section{Correlation between vEEG and mEEG}

The optimal parameters determined on the train set resulted in a mean correlation of 0.57 and were fixed for evaluation on the test set. When applying the processing chain with the fixed parameters to the test set, the resulting mean correlation is 0.64 . The volume of data left for the analysis after artifact removal equals $73 \%$ of the original data volume.

In order to better understand the distribution of correlations, each patient was analyzed individually (Fig. 8). While most patients produce similar results, some patients have much lower overall correlation coefficients.

The results are highly sensitive to the choice of the frequency band. Considering the fact that the bands of interest might differ depending on the application, the correlations were examined independently for each frequency band (Tab. 2). The alpha band shows the highest correlation ( 0.74 on the test set).

\section{Correlations between vEEG electrodes}

In order to obtain the comparison correlation values, the correlation between neighboring video electrodes, positioned at the distance larger or equal to 3 from G2 were computed and averaged on the full data set. The received grand average was 0.78 (see Fig. 9 for details). The corresponding average on full data set for similarly distant (3 to 4 ) mobile-to-video quadruples was 0.57 . To compare the quality of the mEEG and vEEG, we report the percentage of data retained during cleaning for both mEEG to vEEG and vEEG to vEEG correlations (Tab. 3). The vEEG to vEEG comparison retains approx. $89-96 \%$ of the data, which means that $4-11 \%$ are discarded. In the mEEG to vEEG comparison, approx. $26-28 \%$ of the data are discarded, which indicates that the mEEG is responsible for $15-24 \%$ or two thirds of the total discarded data. 


\section{Discussion}

\section{Main Findings}

408 The main goal of this work was to find a way to objectively (quantitatively) validate a mobile

409 EEG device in a situation when only resting state brain waves can be collected and no hardware

410 manipulations can be performed (e.g., due to regulations). In particular, the positions of the

411 individual electrodes are fixed and no common referencing is available. To overcome these

412 limitations, we have developed an advanced and multi-parametric data processing procedure,

413 which allows obtaining illustrative and robust results in the form of a grand average correlation

414 coefficient.

415 The resulting average correlation is dependent on the chosen frequency range. In case of EEG

416 data it is informative to consider standard brain wave frequency bands (delta, theta, alpha and

417 beta). The average correlation varies from 0.62 on the delta band of the test set to 0.74 on alpha

418 band (on the test set). The average across the overall considered frequency band $(1-38 \mathrm{~Hz})$ is

419 0.64. While this number may look moderate, it can be percepted as quite high if we take into

420 account that as much as $73 \%$ of all data are preserved and that we compute a grand average over

421 multiple (on average 19 per patient) quadruples of $30 \mathrm{~min}$ long data vectors.

422 For the illustrational purposes we refer to Fig. 10, where two bipolar signals from one quadruple

423 are visibly similar, the quality of the data looks good, but the correlation coefficient is still

424 "only" 0.72.

425 Due to chance, the average correlation on the test set is higher than on the train set. One of the main reasons is a consistency in signal quality within one record and relatively small $(n=19)$

427

428

429

430

431

432

433

434

435

436

437

438

439

440

441

442

443 number of records, resulting in only 5 test records. Nevertheless, both train and test sets show similarity in the results and have similar dynamics across the bands, with alpha band showing the highest correlation.

Although the overall data quality was not optimal, only three out of 22 sessions were rejected because of recording failures, but none during the processing pipeline execution. For the remaining 19 sessions, on average, 19 quadruples of electrodes were selected based on strictly formulated criteria. Furthermore, only $27-28 \%$ of data were lost due to rejection of full channels or signal segments (train and test sets). The rejection was performed only based on computer algorithms. Thereby, the reported correlation averages across multiple session and quadruples, and covers most of the recorded data. With respect to the high amount of data and potential variation, the low standard deviation of 0.14 (on test set) points towards high robustness.

Due to the uniqueness of the data it is a difficult task to evaluate the results. Therefore we have performed a test to relate the grand average correlation. The average correlation was computed for neighbouring pairs of vEEG electrodes and resulted in a value of 0.78 , which lies half way between vEEG-mEEG correlation on "medium" quadruples (0.57) and perfect correlation of 1 . 
444 The assumptions regarding possible deviations from a perfect correlation listed above are 445 difficult to verify, so we can only hypothesize that these values suggest lower signal quality of 446 mEEG comparing to the clinical device, but the differences seem to be moderate.

\section{Comparison to the state of the art}

448 Analyzing other works on electrodes quality comparison, where experiments were performed in

449

450

451

452

453

454

455

456

457

458

459

460

461

462

463

464

465

466

467

468

469

470

471

472

473

474

475

476

477

478

479

480

481

482 a more controlled environment, may bring a better understanding of how much of the signal differences are caused by spatial shift of the electrodes and how much by the lower quality of the mobile hardware. Only experiments of "same-time-different-place" type with resting state or similar conditions were chosen for the comparison. In some papers, the authors compare the same type of electrodes in order to estimate the spatial shift-related signal change.

In Fiedler et al. (6) the authors place 3 types of dry electrodes at Fp1, Fp2, O1 and O2 sites, with standard wet electrodes adjacent. Additionally two sets of wet electrodes was tested to provide a baseline. In resting state the resulting average correlations were: $0.24,0.59$, and 0.25 for three dry-to-wet comparisons respectively and 0.58 for wet-to-wet combination.

Estepp et al. (5) under the open eyes condition reported $0.84,0.61$ and 0.32 for dry-to-wet comparison at $\mathrm{Fz}, \mathrm{C} 4$ and $\mathrm{Pz}$ positions respectively. Similarly, wet-to-wet combinations resulted in $0.97,0.95$ and 0.80 .

In Wyckoff et al. (2) the measurements with dry and wet electrodes were done at $\mathrm{Fz}, \mathrm{C} 3, \mathrm{Cz}, \mathrm{C} 4$ and Pz. For the open eyes condition the average correlation varied from 0.28 on delta band to 0.99 on alpha, beta $1(13-16 \mathrm{~Hz})$ and beta $2(13-21 \mathrm{~Hz})$ bands.

Liao et al. (1) reported respectively 0.95 and 0.91 correlation at F10 and POz for two different electrode types.

More references can be found in a review paper of Lopez-Gordon et al. (3). The results in the above referenced papers are characterized by high variability. The reported correlations vary from 0.25 to 0.97 on time-domain signal, which can be explained by different quality of the tested electrodes, but also by differences in the placement, experimental details and data processing. All the experiments were carefully controlled, electrode number limited to a maximum of 5 , subjects movement could be minimized and sometimes the segments of data rejected after visual examination (4).

In contrast, in our research, the data were collected under minimal control, multiple electrode sites were used (including the ones known for high artifact presence) and no human examination was used for data processing. Yet, the results are revealing a correlation level comparable to other comparisons with a similar setup.

Peer] reviewing PDF | (2019:10:42184:1:1:NEW 2 Mar 2020) 


\section{Limitations}

484 In the described work Pearson correlation was used as a straightforward similarity measurement.

485 It seems to be a reasonable choice in the given context, as linear relationship is exactly what we

486 are expecting from our experiments if the spatial shift is neglected. On the other hand, this spatial

487 shift may introduce significant nonlinear effects. In the further work it might be beneficial to

488 consider different measurements of similarity, such as mutual information (9). Nonlinear

489 relationships in EEG have been deeply studied in the context of epileptic seizures where

490 synchronisation of different brain areas often occurs. In Quiroga et al. (8) a number of nonlinear

491 measurements is discussed, however the same paper also suggests ultimate resulting similarity of

492 the different types of measurements, including linear correlation.

493 Another limitation is the restriction of the comparison bandwidth from 1 to $38 \mathrm{~Hz}$. The maximum

494 available frequency of our setup is 64, however only little mutual information was detectable in

495 the higher frequencies. This indicates both a limitation in the hardware, as well as in the

496 proposed algorithm, as the parts with potentially worse correlation are excluded. While this is

497 reasonable as long as the values chosen are still clinically relevant, it requires special care when

498 choosing the $f q \_$lowpass value.

\section{Conclusion}

500 In this work we have developed a data analysis procedure designed to deal with two sources of

501 EEG data recorded simultaneously during resting state. This procedure aims to provide an

502 objective measurement of the data quality.

503 It is not uncommon that in the procedure of EEG comparison visual assessment by the trained

504 specialists is used as a part of data pre-processing (4). While professional opinion may provide a

505 unique insight, it is also very costly to obtain. In the particular case of our study, more than 20

506 hours of multichannel data need to be analyzed. Moreover, multiple studies have shown that

507 human assessment is not fully reproducible and high intra- and inter-rater variances are

508 consistently reported $(18,19)$, which could be reproduced on our data in a previous publication

509 (12). In contrast, automated analysis pipeline provides an objective, fast and low-cost way to

510 perform the data comparison.

511 The presented procedure deals with the challenges of different referencing, spatial shifting of the

512 electrodes and lack of controlled stimuli (such as in ERP experiments). Using automatically

513 optimized parameters for the pre-processing, a grand average linear correlation of 0.64 between

514 mobile and clinical EEG devices was obtained. It was compared to several baseline correlations,

515 such as clinical-to-clinical EEG correlation, to conclude that the overall quality of the considered

516 mobile device is good, since similar correlations can be seen when only electrode types are

517 changed (e.g. when comparing wet and dry electrodes). This result agrees with our previous

518 study, where trained neurologists were clinically investigating the data (12), and with a number

519 of other studies done on Epoc Emotiv in different contexts $(11,20)$. However, to our best 
520 knowledge, a fully automated approach in combination with the resting state data was not 521 previously reported.

522 The presented pipeline might benefit in the future from including more sophisticated signal 523 processing methods, such as mutual information. Nevertheless, in the current form it already 524 shows high efficiency and might be potentially generalizable to different multi-channel sensors, 525 such as EMG or ECG.

\section{Acknowledgements}

527 The authors are grateful to Jonas Schulte-Coerne for valuable discussions on the signal

528 processing and to prof. Peter König for a very insightful revision, which significantly improved 529 the paper quality.

\section{References}

531

532

533

534

535

536

537

538

539

540

541

542

543

544

545

546

547

548

549

550

551

552

553

554

555

556

557

558

559

1. Liao L-D, Wang I-J, Chen S-F, Chang J-Y, Lin C-T. Design, Fabrication and Experimental Validation of a Novel Dry-Contact Sensor for Measuring Electroencephalography Signals without Skin Preparation. Sensors. 2011 May 30;11(6):5819-34.

2. Wyckoff SN, Sherlin LH, Ford NL, Dalke D. Validation of a wireless dry electrode system for electroencephalography. J NeuroEngineering Rehabil [Internet]. 2015 Oct 31 [cited 2018 May 25];12. Available from: https://www.ncbi.nlm.nih.gov/pmc/articles/PMC4628242/

3. Lopez-Gordo M, Sanchez-Morillo D, Valle F. Dry EEG Electrodes. Sensors. $2014 \mathrm{Jul}$ 18;14(7):12847-70.

4. Gargiulo G, Calvo RA, Bifulco P, Cesarelli M, Jin C, Mohamed A, van Schaik, A. A new EEG recording system for passive dry electrodes. Clin Neurophysiol. 2010 May;121(5):68693.

5. Estepp JR, Christensen JC, Monnin JW, Davis IM, Wilson GF. Validation of a Dry Electrode System for EEG. Hum Factors Ergon Soc Annu Meet Proc. 2009 Oct 19;53(18):1171-5.

6. Fiedler P, Haueisen J, Jannek D, Griebel S, Zentner L, Vaz F, Fonseca C. Comparison of three types of dry electrodes for electroencephalography. ACTA IMEKO. 2014 Sep 23;3(3):33.

7. Grozea C, Voinescu CD, Fazli S. Bristle-sensors-low-cost flexible passive dry EEG electrodes for neurofeedback and BCI applications. J Neural Eng. 2011;8(2):025008.

8. Quian Quiroga R, Kraskov A, Kreuz T, Grassberger P. Performance of different synchronization measures in real data: a case study on electroencephalographic signals. Phys Rev E Stat Nonlin Soft Matter Phys. 2002 Apr;65(4 Pt 1):041903.

9. Mikkelsen KB, Kidmose P, Hansen LK. On the Keyhole Hypothesis: High Mutual Information between Ear and Scalp EEG. Front Hum Neurosci [Internet]. 2017 Jun 30 [cited 2018 May 25];11. Available from: http://journal.frontiersin.org/article/10.3389/fnhum.2017.00341/full

10. Badcock NA, Preece KA, de Wit B, Glenn K, Fieder N, Thie J, McArthur G. Validation of the Emotiv EPOC EEG system for research quality auditory event-related potentials in children. PeerJ. 2015;3:e907.

Peer) reviewing PDF | (2019:10:42184:1:1:NEW 2 Mar 2020) 
560

561

562

563

564

565

566

567

568

569

570

571

572

573

574

575

576

577

578

579

580

581

582

583

584

585

586

587

588

589

590

591

592

593

594

595

596

597

598

599

600

11. Melnik A, Legkov P, Izdebski K, Kärcher SM, Hairston WD, Ferris DP, König P. Systems, Subjects, Sessions: To What Extent Do These Factors Influence EEG Data? Front Hum Neurosci [Internet]. 2017 Mar 30;11. Available from: http://www.ncbi.nlm.nih.gov/pmc/articles/PMC5371608/

12. Titgemeyer Y, Surges R, Altenmüller DM, Fauser S, Kunze A, Lanz M, Malter MP, Nass RD, von Podewils F, Remi J, von Spiczak S, Strzelczyk A, Ramos R, Kutafina E, Jonas S. Can commercially available wearable EEG devices be used for diagnostic purposes? An explorative pilot study. Epilepsy \& Behavior. 2019 Oct 19.

13. Tatum WO. Handbook of EEG interpretation [Internet]. New York [N.Y.: Demos Medical Pub.; 2013 [cited 2018 Oct 8]. Available from: http://www.credoreference.com/book/spheegi

14. Fink I. Mobile EEG Presentation Framework [Bachelor Thesis]. [Aachen, Germany]: RWTH Aachen University; 2016.

15. Delorme A, Makeig S. EEGLAB: an open source toolbox for analysis of single-trial EEG dynamics including independent component analysis. J Neurosci Methods. 2004 Mar 15;134(1):9-21.

16. Ball T, Demandt E, Mutschler I, Neitzel E, Mehring C, Vogt K, Aersten A, SchulzeBonhage A. Movement related activity in the high gamma range of the human EEG. NeuroImage. 2008 Jun;41(2):302-10.

17. Darvas F, Scherer R, Ojemann JG, Rao RP, Miller KJ, Sorensen LB. High gamma mapping using EEG. NeuroImage. 2010 Jan 1;49(1):930-8.

18. Grant AC, Abdel-Baki SG, Weedon J, Arnedo V, Chari G, Koziorynska E, Lushbougha C, Mausa D, McSweena T, Mortatia KA, Reznikova A, Omurtag A. EEG interpretation reliability and interpreter confidence: a large single-center study. Epilepsy Behav EB. 2014 Mar;32:102-7.

19. Benbadis SR. Generalized Epilepsies on EEG: Overview, Types of Waveforms Associated With Generalized Epilepsies, Clinical Presentation. 2017 Mar 30 [cited 2017 Apr 24]; Available from: http://emedicine.medscape.com/article/1140724-overview

20. McKenzie ED, Lim ASP, Leung ECW, Cole AJ, Lam AD, Eloyan A, Nirola DK, Tshering L, Thibert R, Zepeda Garcia R, Bui E, Deki S, Lee L, Clark SJ, Cohen JM, Mantia J, Brizzi KT, Sorets TR, Wahlster S, Borzello M, Stopczynski A, Cash SS, Mateen FJ. Validation of a smartphone-based EEG among people with epilepsy: A prospective study. Sci Rep. 2017 Apr 3;7:srep45567.

21. Zschocke S, Kursawe H, editors. Klinische Elektroenzephalographie, Springer Medizin; 2012. $656 \mathrm{p}$.

\section{Appendix}

Table A1 presents the median time lags between mobile and video bipolar signals. The signals were divided into 4 approximately equal length vectors in order to track the possible drift of the delay in time. The median is chosen to avoid the influence of the outliers, which are present due to bad quality of the data in some channels. In this case the lags computed based on autocorrelation are not informative. 
Figure 1

Example of the VEEG (green) and mEEG (blue) electrode placement.

(A) The placements differ slightly from patient to patient, therefore a sketch is made for each session to track the relative electrode positioning. (B) Zoom to an example electrode quadruple.

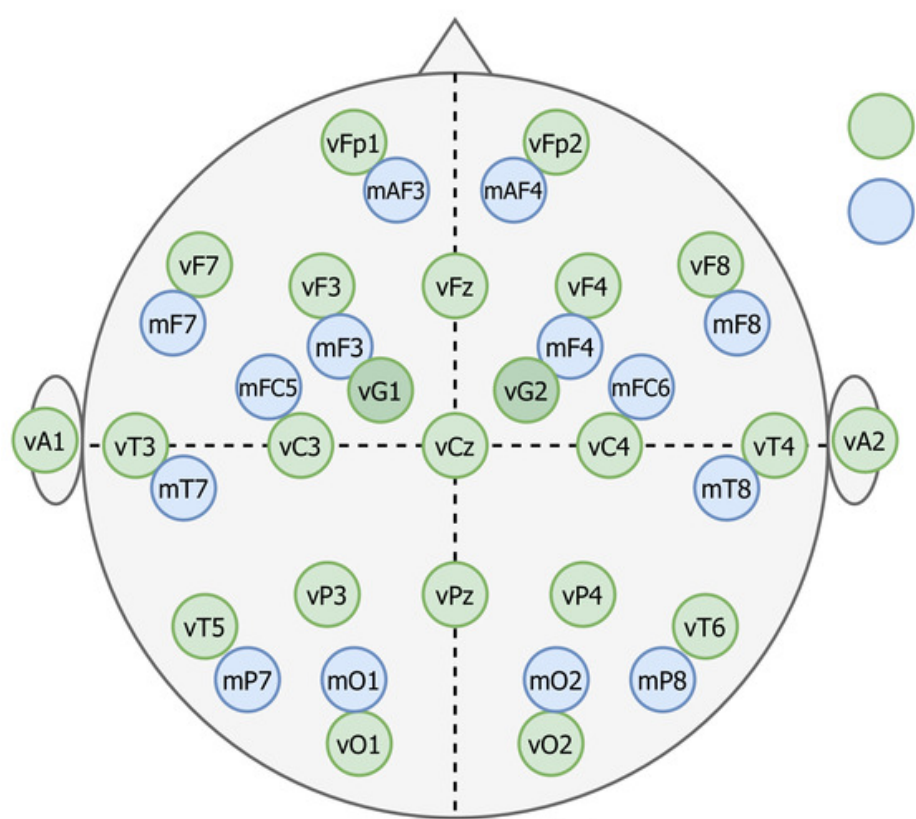

(a)
Video EEG

EPOC Mobile EEG
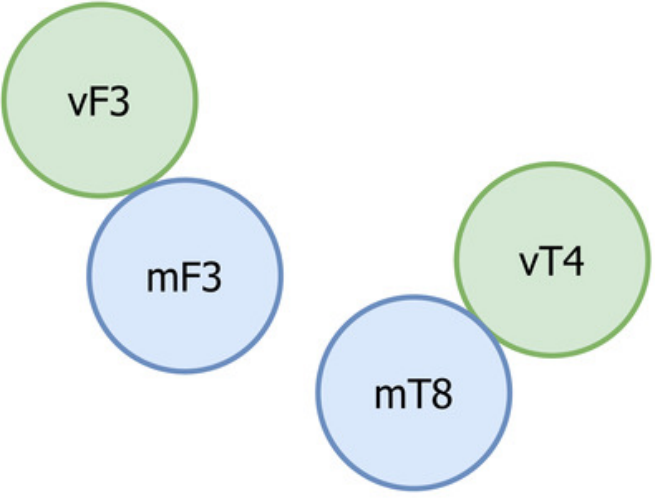

(b) 


\section{Figure 2}

Fragment of EEG record with spike-slow wave abnormalities.

(a) vEEG. (b) mEEG.

(a)

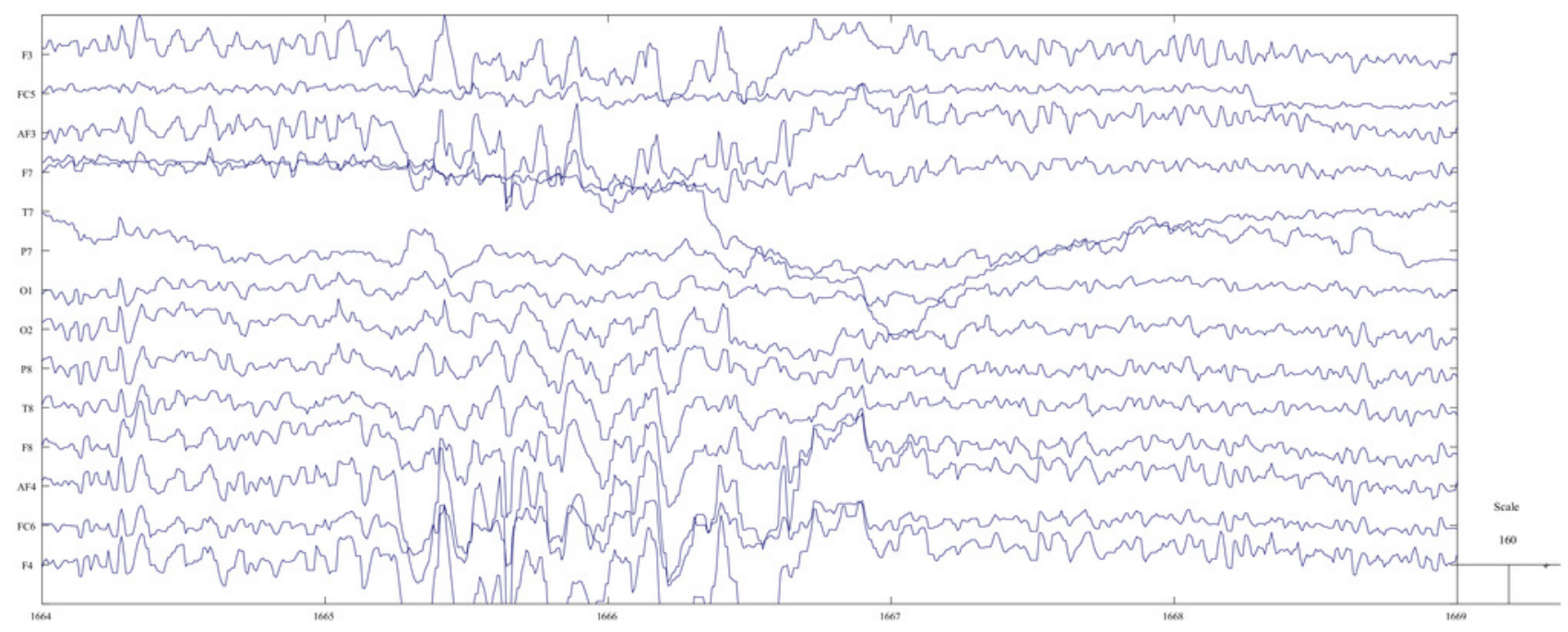

(b)

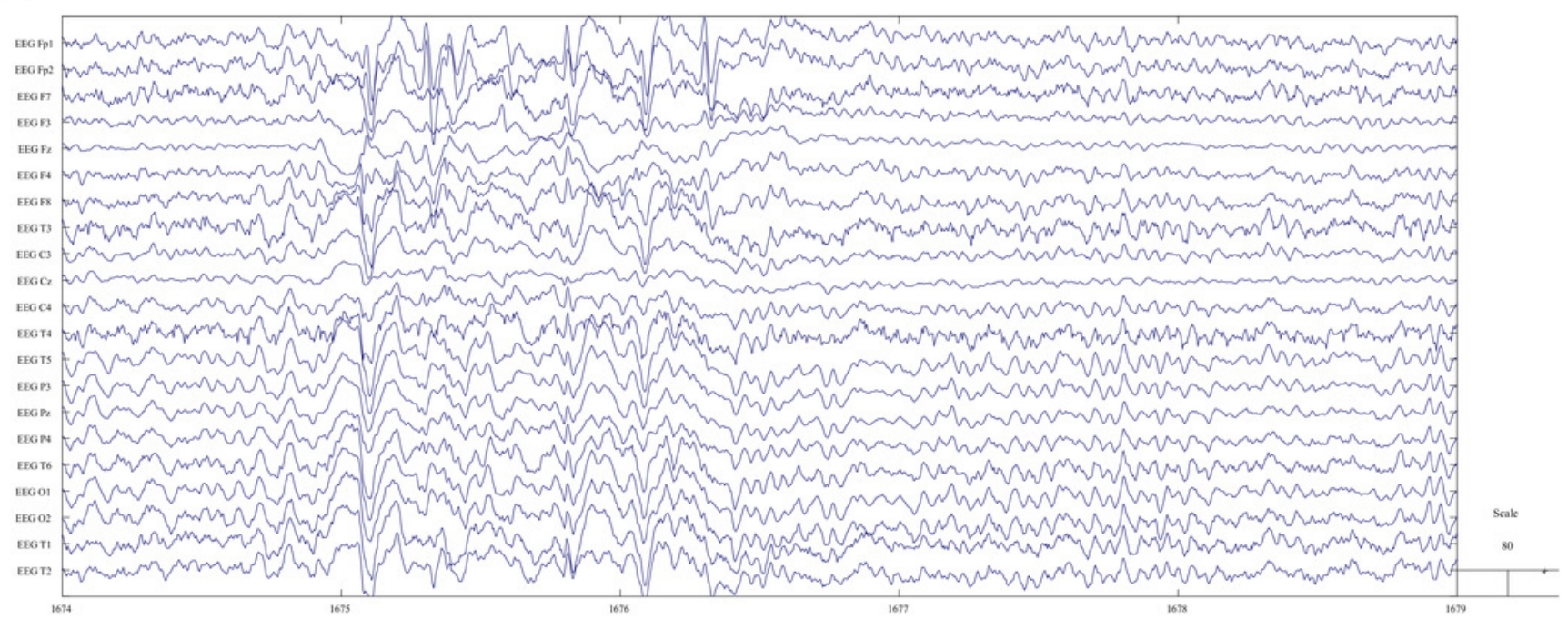


Figure 3

Initial pruning and quadruplet list creation.

Using timestamps and blinking artifacts approximately simultaneous vEEG and mEEG files are obtained. Personal sketch of electrode positions allows to detect spatially close electrode pairs and generate reference-free quadruples.

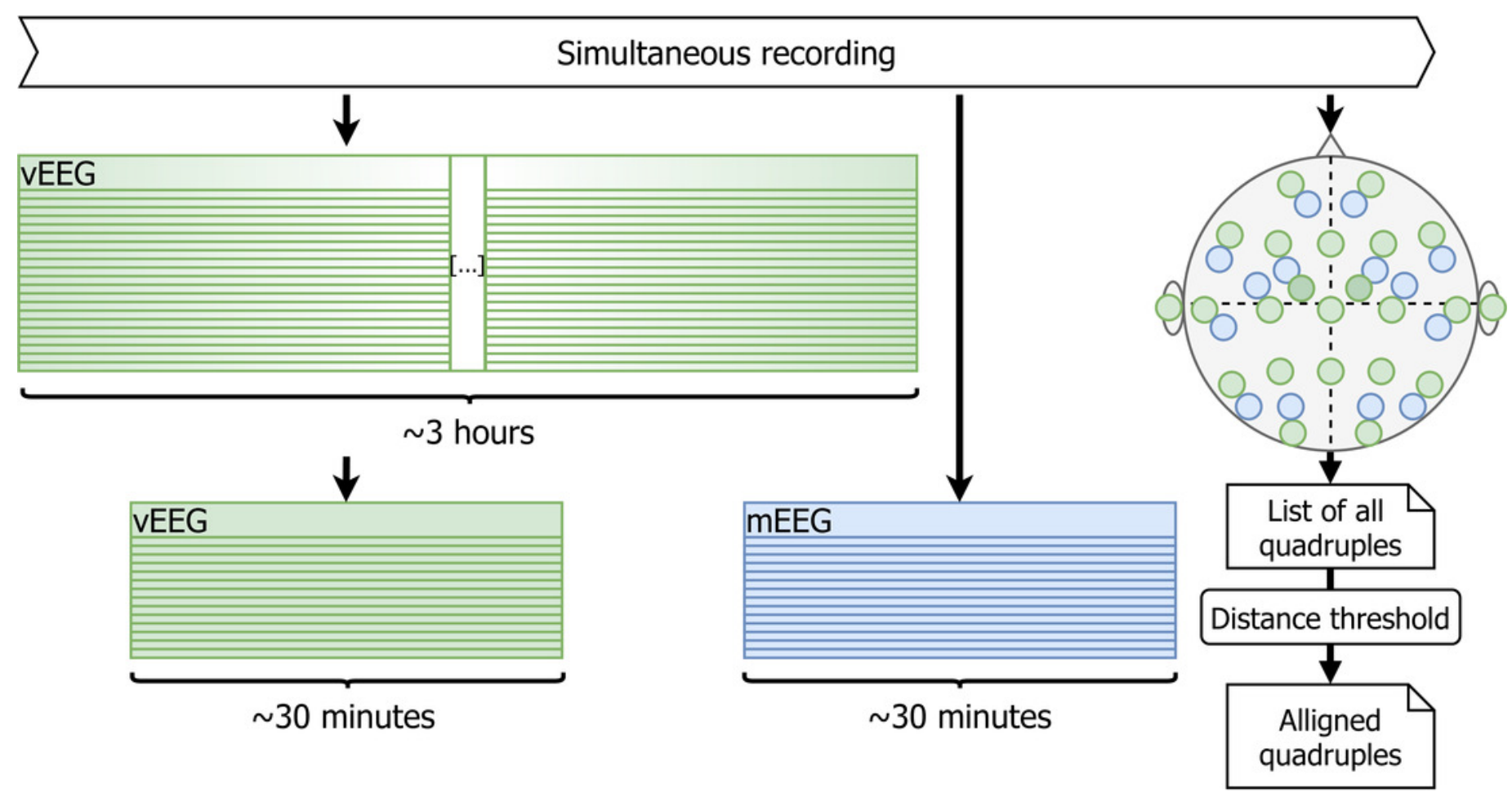


Figure 4

VEEG electrodes put on an approximate grid.

The grid allows to compute Manhattan distances between different electrodes. Reference electrode $\mathrm{G} 2$ is placed in the middle of the unit square to reflect the realistic placement.

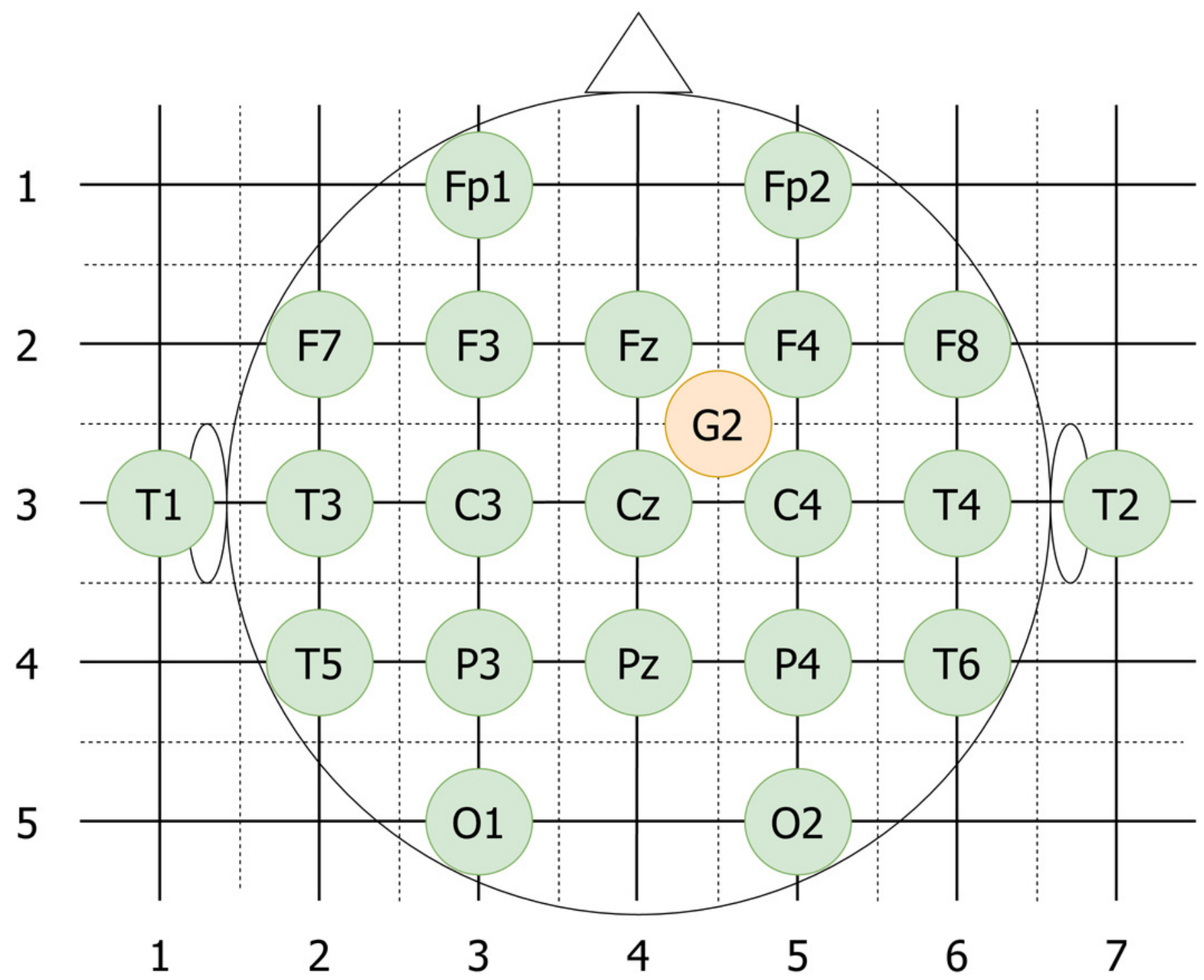


Figure 5

Data cleaning.

Data cleaning involves removal of corrupted channels (followed by the update of the quadruple list), filtering, resampling and normalization of the data.
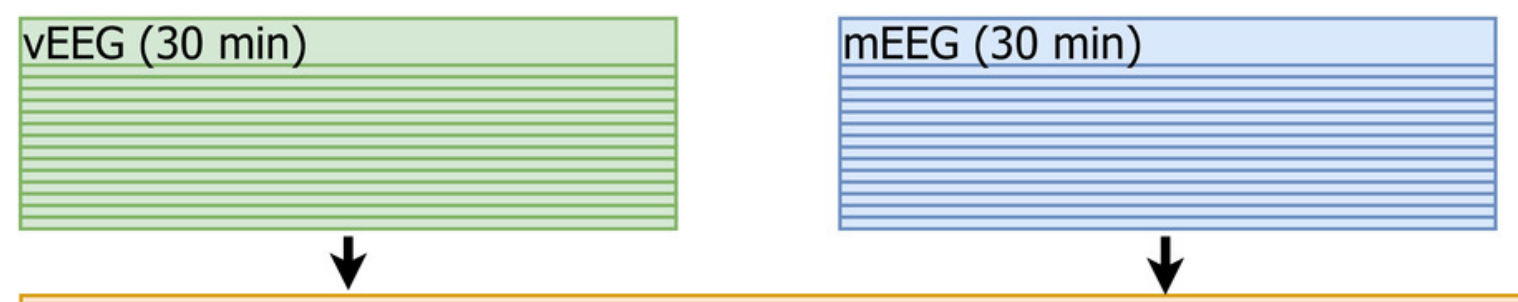

List of quadruplets

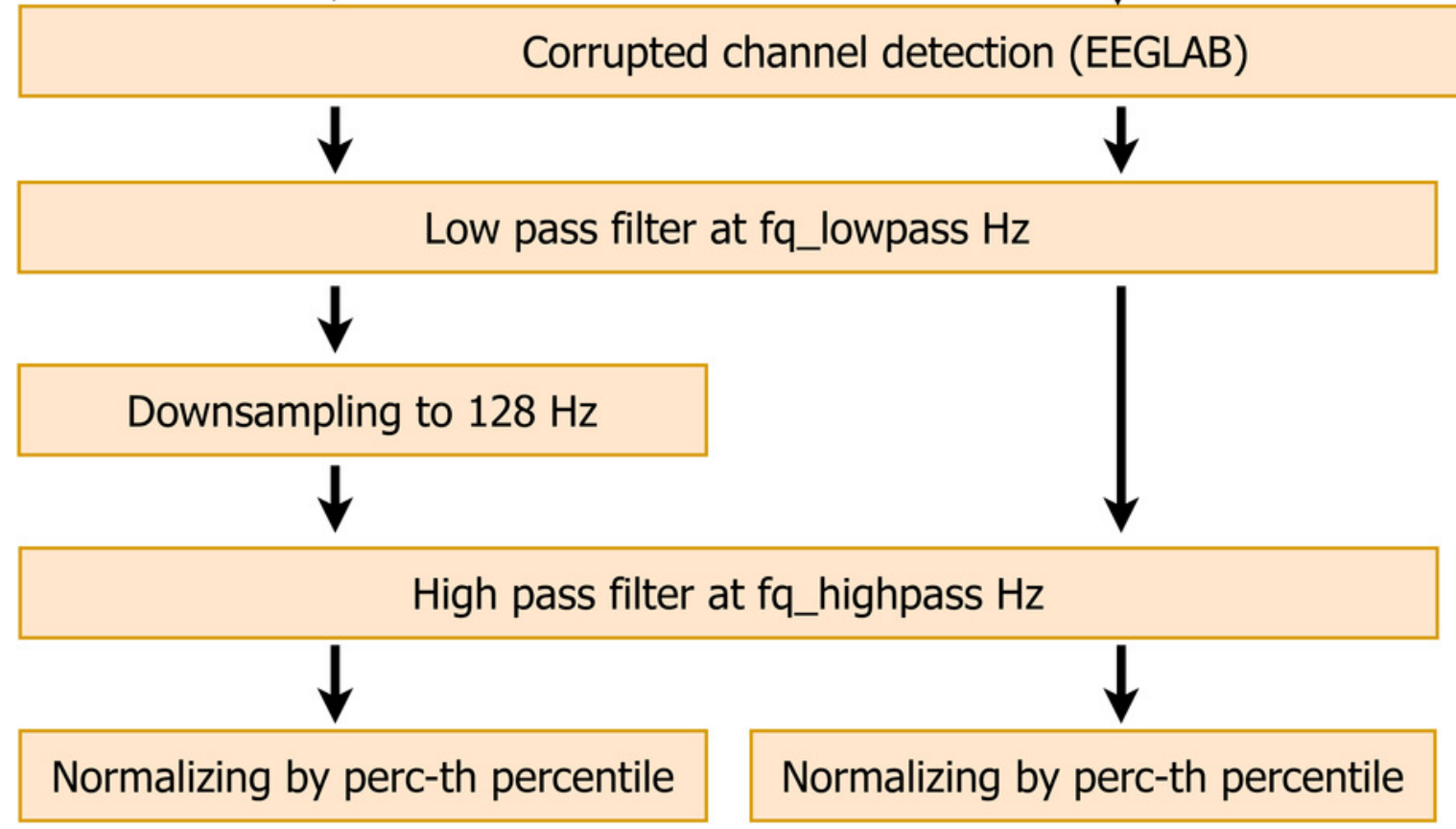


Figure 6

Pipeline for creating matching data vectors to compare through linear correlation.

For each quadruple artifact detection is performed in each electrode's data, then time aligned reference free vectors are constructed and the artifacts are removed without breaking the time synchronisation. Vectors with low signal-to-noise ratio are removed from the list. Linear correlation is computed for the remaining matching vectors.

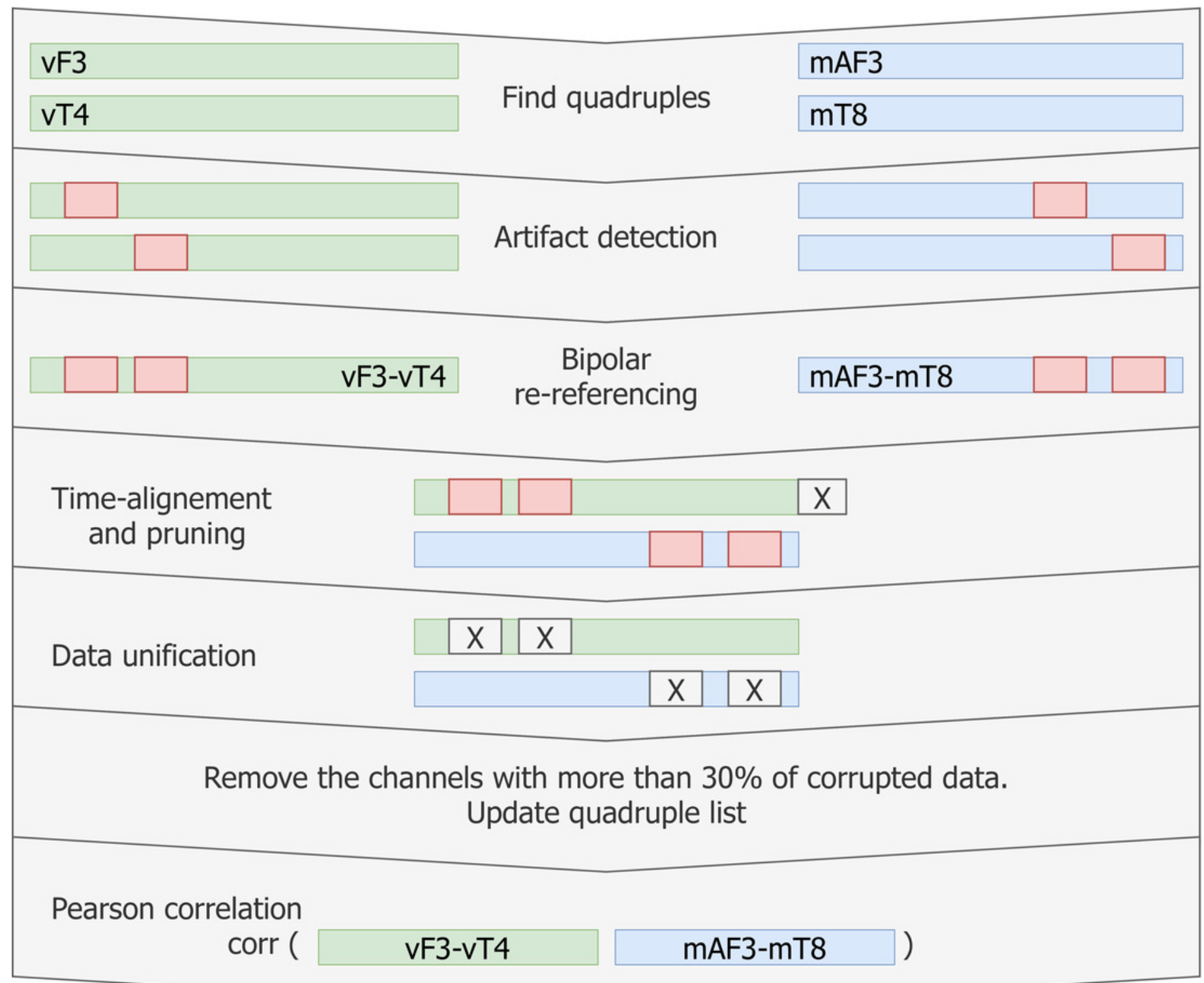


Figure 7

Magnitude-squared coherence between mEEG and vEEG averaged across electrodes and patients.

Red lines indicate thresholds for fq_highpass and fq_lowpass.

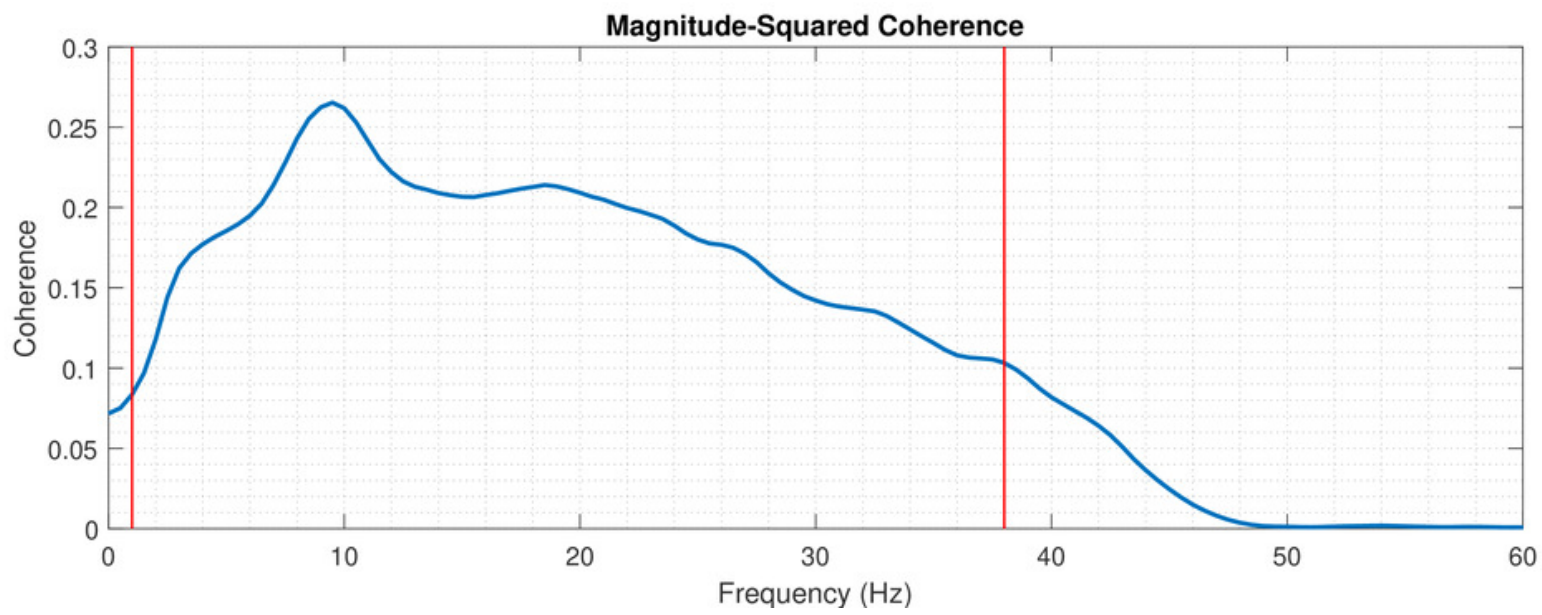


Figure 8

Distribution of correlations for single quadruples per individual patient.

Results on the test set are shown in red and on the train set in blue.

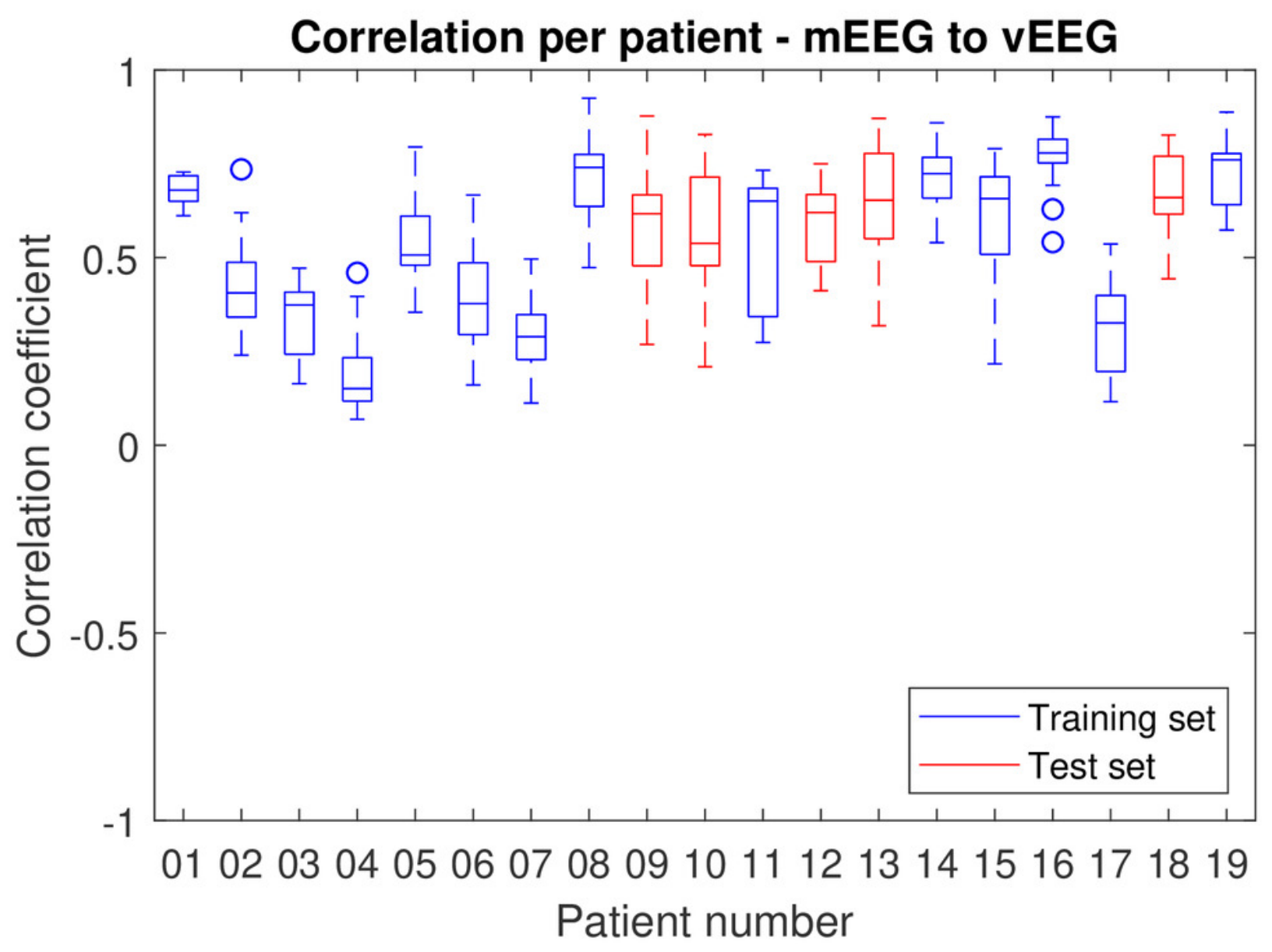


Figure 9

Distribution of correlations between pairs of VEEG electrodes over individual patients.

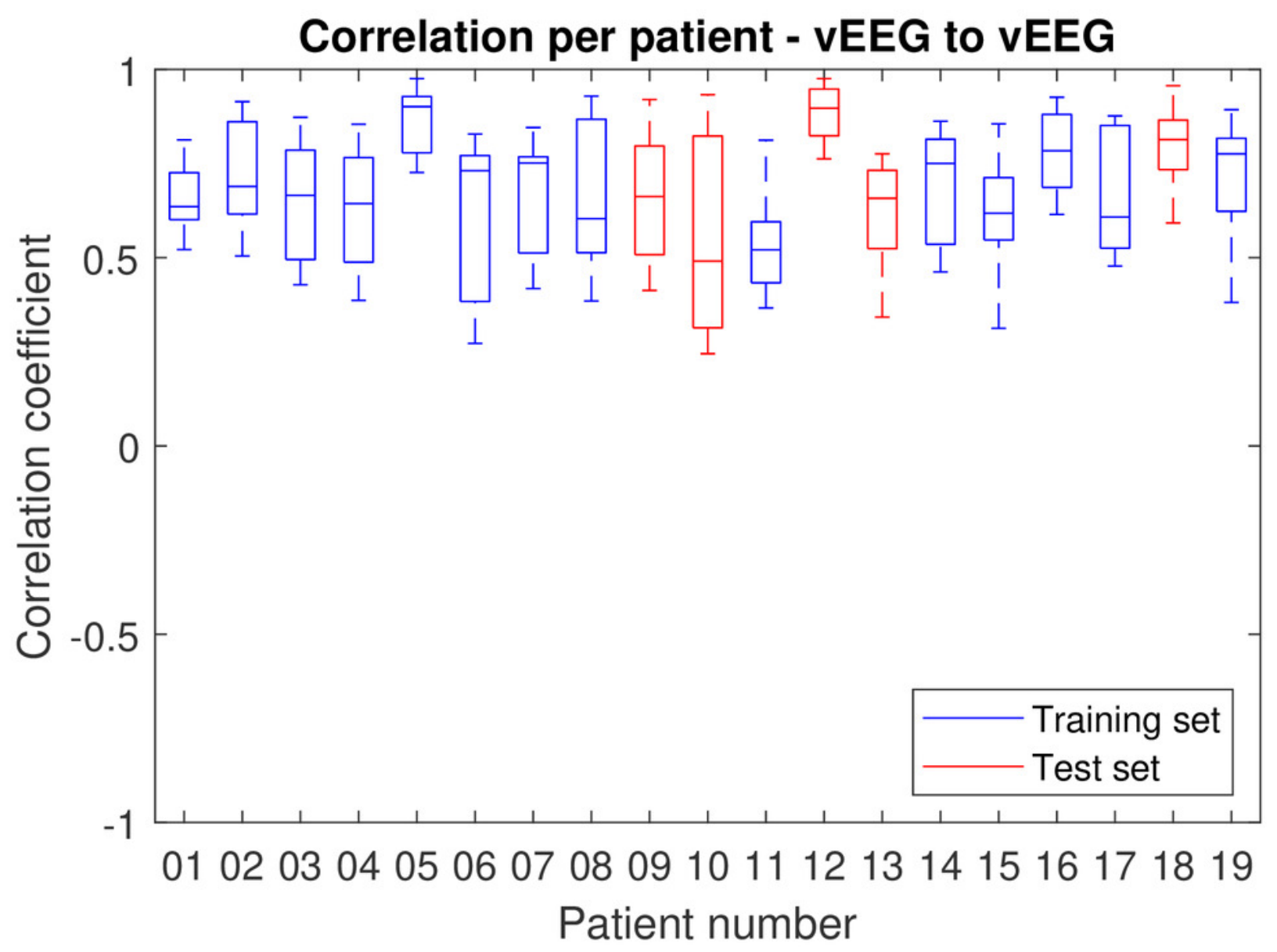


Figure 10

Fragment of two bipolar signals from one quadruple.

The signals are processed according to the described pipeline and therefore well aligned. The correlation between the illustrated fragments equals 0.72 .

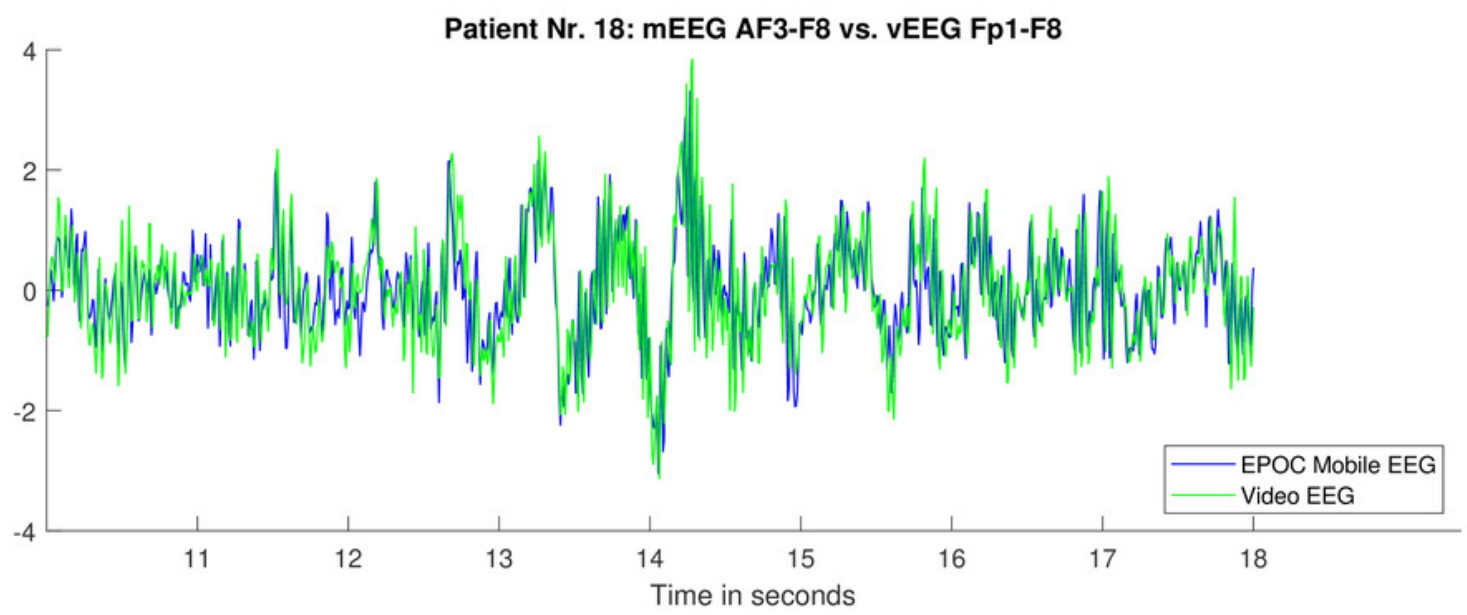


Table $\mathbf{1}$ (on next page)

Demographic data of the participants. 


\begin{tabular}{|c|c|c|}
\hline & Count & $\%$ \\
\hline \multicolumn{3}{|l|}{ Sex } \\
\hline Male & 11 & $50 \%$ \\
\hline Female & 11 & $50 \%$ \\
\hline \multicolumn{3}{|l|}{ Age } \\
\hline$<30$ & 6 & $27,2 \%$ \\
\hline $30-40$ & 5 & $22,7 \%$ \\
\hline $40-50$ & 5 & $22,7 \%$ \\
\hline$>50$ & 6 & $27,2 \%$ \\
\hline
\end{tabular}

2 


\section{Table 2 (on next page)}

Average correlation by frequency band for the test set group.

Gamma is not analyzed individually as only part of the band is present in the signal. 
1

\begin{tabular}{|l|l|l|l|l|l|}
\hline $\begin{array}{l}\text { Average } \\
\text { correlation }\end{array}$ & \multicolumn{1}{|c|}{$\begin{array}{c}\text { Delta } \\
(1-3.5 \mathrm{~Hz})\end{array}$} & $\begin{array}{c}\text { Theta } \\
(3.5-7.5 \mathrm{~Hz})\end{array}$ & $\begin{array}{c}\text { Alpha } \\
(7.5-12.5 \mathrm{~Hz})\end{array}$ & $\begin{array}{c}\text { Beta } \\
(12.5-30 \mathrm{~Hz})\end{array}$ & $\begin{array}{c}\text { Full band } \\
(1-38 \mathrm{~Hz})\end{array}$ \\
\hline Train set & 0.51 & 0.62 & $\mathbf{0 . 6 8}$ & 0.54 & 0.57 \\
\hline Test set & 0.62 & 0.73 & $\mathbf{0 . 7 4}$ & 0.64 & 0.64 \\
\hline Full data set & 0.55 & 0.66 & $\mathbf{0 . 7 0}$ & 0.57 & 0.60 \\
\hline
\end{tabular}

2

3 
Table 3 (on next page)

Percentage of data left after channel rejection and artifact removal. 
1

\begin{tabular}{|l|l|l|l|}
\hline Data left in total (in \%) & \multicolumn{1}{|c|}{ Test set } & \multicolumn{1}{|c|}{ Train set } & \multicolumn{1}{c|}{ Full data set } \\
\hline $\begin{array}{l}\text { mEEG - vEEG (,long } \\
\text { distances“) }\end{array}$ & 72.83 & 71.67 & 71.98 \\
\hline $\begin{array}{l}\text { mEEG - vEEG } \\
(, \text { medium distances“) }\end{array}$ & 73.21 & 73.60 & 73.31 \\
\hline vEEG - vEEG & 95.71 & 89.17 & 90.89 \\
\hline
\end{tabular}

2

3 


\section{Table 4 (on next page)}

Median (computed across quadruples) lag between video and mobile EEG signals.

The signal length is divided into 4 equal in time parts to track possible drifts. 


\begin{tabular}{|l|r|r|r|r|}
\hline Patient & Median lag $1^{\text {st }}$ quarter & Median lag $2^{\text {st }}$ quarter & Median lag $3^{\text {st }}$ quarter & Median lag $4^{\text {st }}$ quarter \\
\hline 1 & 739 & 739 & 739 & 739 \\
\hline 2 & 584 & 584 & 584 & 583 \\
\hline 3 & 587 & 587 & 614 & 614 \\
\hline 4 & 780 & 780 & 780 & 780 \\
\hline 5 & 1046 & 1045 & 1045 & 1045 \\
\hline 6 & 493 & 492 & 492 & 492 \\
\hline 7 & 1136 & 1136 & 1136 & 1135 \\
\hline 9 & 1185 & 1185 & 1185 & 1176 \\
\hline 10 & 1106 & 1106 & 1106 & 1105 \\
\hline 11 & 1286 & 1286 & 1286 & 1286 \\
\hline 13 & 1218 & 1218 & 1218 & 1218 \\
\hline 14 & 1240 & 1240 & 1239 & 1239 \\
\hline 15 & 1260 & 1260 & 1260 & 1259 \\
\hline 16 & 1245 & 1245 & 1245 & 1245 \\
\hline 17 & 1275 & 1275 & 1275 & 1275 \\
\hline 18 & 1404 & 1404 & 1403 & 1403 \\
\hline 19 & 1330 & 1331 & 1330 & 1330 \\
\hline 20 & 1350 & 1350 & 1350 & 1350 \\
\hline 21 & 1252 & 1252 & 1252 & 1252 \\
\hline & & & & \\
\hline
\end{tabular}

1

2 